\title{
Detection and interpretation of the Earth's free oscillations excited by the great Sumatra-Andaman earthquake with GGP station data*
}

\author{
Xiang'e Lei` Heping Sun and Houtse Hsu \\ Institute of Geodesy and Geophysics, Chinese Academy of Sciences, Wuhan 430077, China
}

\begin{abstract}
The mode serials of the Earth's free oscillation provide some important information on the Earth's deep structure and superconducting gravimeters (SG) can investigate the phenomena of the Earth's free oscillations with high accuracy. The great Sumatra-Andaman earthquake fully excited the Earth's free oscillations and these signals were perfectly recorded by five superconducting gravimeters in the globe. After the pre-treatment and spectral analysis on the SG observation data, we obtained the experimented mode serials of the Earth's free oscillations consisting of 147 modes with GGP station data. These observed modes were themselves some new important data for the study of the Earth's deep structure. On the basis of the discussions on some checked inner-core-sensitive modes, we distinguished three layers from the inner core, and the boundary of the upper layer was compatible with the formerly known transition zone in the inner core based on seismic body waves and supported that there were the hemispherical variation and very lower shear velocity zone in the lower inner core.
\end{abstract}

Key words: Sumatra-Andaman earthquake; GGP station data; Earth's free oscillation; Earth's inner core; core sensitive mode

CLC number: P315.2 Document code: A

\section{Introduction}

The Earth's free oscillations (EFO) are usually excited after some large earthquakes. In 1961, the EFO phenomenon was first observed separately with the strainmeter (Benioff et al., 1961) and with spring gravimeter (Ness et al., 1961), which opened formally the prologue of academic EFO subject. The EFO phenomena provides a valuable path independent of seismic body waves to investigate the Earth's deep structure (Fu et al., 1985; Garland, 1979; Widmer et al., 1991), by comparing the experimented EFO modes with the theoretical predictions basing on the Earth's known construction. The EFO modes have two basic categories: spheroidal modes and toroidal modes. As the spheroidal modes can propagate in the whole Earth, it

\footnotetext{
* Received 19 September 2010; accepted in revised form 29 November 2010; published 10 April 2011.

* Corresponding author. e-mail: leixe@whigg.ac.cn

(C) The Seismological Society of China and Springer-Verlag Berlin Heidelberg 2011
}

provides a window to look into the Earth's deep construction (Garland, 1979; He and Tromp, 1996). So it is still an important aspect for the EFO research to obtain accurately the experimented mode serials at present.

Derr (1969) investigated the spheroidal modes with spring gravimeters and Dziewonski and Gilbert (1972) presented the classic paper on the observation of EFO modes with the long-period seismographs. Dziewonski and Anderson (1981) provided the famous preliminary reference Earth model (PREM) based on many kinds of observation data. The mode serials of experimented spheroidal EFO are mainly determined by the longperiod seismographs or spring gravimeters before the 1980s. As the superconducting gravimeters (SG) came into being in 1980s, people gained a new kind of modern measure technique to investigate the small vertical deformation of the Earth's surface and the gravity field variation in the period range from seconds to years including the EFO signals with high accuracy. So it is also one of research contents of the Global Geodynamic Project (GGP) to investigate the EFO phenomena 
(Crossley and Hinderer, 1995). Some researchers have studied the EFO phenomena with SG instruments and checked the splitting peaks of the gravest EFO mode with SG observation data (Van Camp, 1999; Neumeyer et al., 2002; Lei et al., 2002a, b, 2007; Rosat et al., 2004, 2005).

The great Sumatra-Andaman earthquake was one of the largest earthquakes since 1964 and fully excited the Earth's free oscillations, which provided a precious chance to study the deep structure of the Earth. We obtained a total of 147 EFO modes with SG instruments at five GGP stations, and they were a new group of observation mode serials of EFO which help to constrain the Earth's deep structure. By the analysis on the checked core-sensitive modes, we presented some complex constructions with three distinct layers and strong hemispherical variation in the inner core, which is compatible with the former observation results obtained by Song and Helmberger (1998) and Sun and Song (2008) based on the travel time data of seismic body wave.

\section{Observation data}

Nearly all SG instruments were produced by the GWR Corporation in USA. The key parts of SG instruments are small superconducting hollow sphere and superconducting coils at the very low temperature shielded from surrounding magnetic field. The small superconducting sphere is always floating in the stable magnetic field produced by the superconducting electric current. If there was a little variation of the gravity field or small deformation of the Earth's surface, the superconducting sphere's position would change and be accurately recorded with an advanced response system. The observation accuracy of SG instruments can arrive at the magnitude of $1.0 \times 10^{-10} \mathrm{~m} / \mathrm{s}^{2}$. The Globe Geodynamic Project (GGP) is an international cooperative project to research all kinds of the geophysical and geodynamic phenomena by employing the continuous and synchronous observations of SG instruments located in the whole globe (Crossley and Hinderer, 1995; Rosat et al., 2004).

The great Sumatra-Andaman earthquake on December 26, 2004 was one of the largest earthquakes since 1964, which not only generated the Indian Ocean tsunami but also excited the EFO. As people are very interested in this large earthquake, many GGP research groups provided two months of the SG data on the GGP website (http://www.eas.slu.edu/GGP/sumatra.html). The SG data files are given in two formats (1 minute and seconds sampling) and listed separately in two directories. We adopted the SG observation data coming from five GGP stations: Wuhan station in China, Canberra station in Australia supported by Japan, Strasbourg station in France, Bad Homburg station and Wettzell station in Germany. As there are two probe spheres for the double sphere SG instruments at Bad Homburg station and Wettzell station, they can provide two groups of recording serials at one SG station. The lower sphere recording is usually the main recording serials with better quality.

The GWR SG C032 was set up in the geodynamic observation station of the Jiufeng Mountain nearby Wuhan city in 1997, which belongs to Institute of Geodesy and Geophysics, Chinese Academy of Sciences. The observational data were recorded by the instrument depending on a set of highly accurate digital collection systems and with the sampling time signals provided by GPS clock. The sampling ratio is 10 seconds for both gravity values and the pressure values (Lei et al., 2002a). The SG C032 was calibrated by the same address observation of several absolute gravimeters (FG5). The calibration value of SG C032 is $-84.6550 \times 10^{-8} \mathrm{~m} \cdot \mathrm{s}^{-2} / \mathrm{V}$ (Sun et al., 2001). Although the calibration and sampling of the other four SG stations should make use of the similar method and technique, the calibration and sampling ratio is still different at different SG station. Some information on the five GGP stations are listed in Table 1, which include the positions, height, instrumental

Table 1 Some information on five GGP stations

\begin{tabular}{|c|c|c|c|c|c|c|c|}
\hline Station & Lat. & $\begin{array}{l}\text { Long. } \\
/{ }^{\circ} \mathrm{E}\end{array}$ & $\begin{array}{l}\text { Height } \\
/ \mathrm{m}\end{array}$ & $\begin{array}{l}\text { SG model } \\
\text { (GWR) }\end{array}$ & $\begin{array}{c}\text { Cal. of SG } \\
/\left(10^{-8} \mathrm{~m} \cdot \mathrm{s}^{-2} \cdot \mathrm{V}^{-1}\right)\end{array}$ & $\begin{array}{l}\text { Cal. of pressure } \\
\qquad /\left(\mathrm{hPa} \cdot \mathrm{V}^{-1}\right)\end{array}$ & $\begin{array}{l}\text { Sampling } \\
\text { ratio/s }\end{array}$ \\
\hline Canberra & $35.32^{\circ} \mathrm{S}$ & 149.01 & 762.75 & $\mathrm{C} 031$ & -75.792 & 1.0 & 60 \\
\hline Strasbourg & $48.62^{\circ} \mathrm{N}$ & 7.68 & 180.00 & $\mathrm{C} 026$ & -79.200 & 22.2222 & 60 \\
\hline Bad Homburg & $50.23^{\circ} \mathrm{N}$ & 8.61 & 190.0 & CD030* & -73.95 & 1.0 & 60 \\
\hline Wettzell & $49.14^{\circ} \mathrm{N}$ & 12.88 & 613.7 & CD029* & -77.366 & 1.0 & 60 \\
\hline Wuhan & $30.52^{\circ} \mathrm{N}$ & 114.49 & 80 & $\mathrm{C} 032$ & -84.6550 & 557.254 & 20 \\
\hline
\end{tabular}

Note: Both CD030 and CD029 are the double spheres superconducting gravimeters and this table presents these information on the lower sphere of SG. 
models, sampling ratio, and the calibration values of SG instruments and the calibration values of pressure recordings.

\section{Pretreatment of observation data}

The great Sumatra-Andaman earthquake gave out so huge energy that the EFO modes were fully excited, which were well recorded by strainmeters, long-period seismographs and superconducting gravimeters (Stein and Okal, 2005; Park et al., 2005). We investigated the EFO phenomena excited by the large earthquake with the observation data of five SG instruments. As EFO modes usually last several days, the influence of some long-period term variations such as polar migration may be neglected. In order to investigate the EFO signals recorded by SG instruments, the pre-treatment process consists of three parts: the correction of gravity tides, the correction of air pressure effect and the appraisal of observational background noise.

The theoretical method is the computation of synthetic tides based on accurate tidal factors for the correction of the gravity tides, however it needs several years of observation data to determine the tidal parameters (Tamura, 1987; Xi, 1998; Xu et al., 2000). So the former researchers (Van Camp, 1999; Neumeyer et al., 2002) usually removed the tidal signals from the observational data with digital filters instead of the computation of synthetic tides. Considering some unsatisfied frequency-phase characteristics of digital filters, we applied a kind of fragmentally polynomial fitting method to the removal of gravity tides (Lei et al., 2002a). The process includes two steps of polynomial fittings, the first step fitting is of 20-class polynomial and the second step is of 10-class polynomial. The optimal data length is about a half day for the fragmental polynomial fitting. After the correction of gravity tides, the tide signals have been basically eliminated from the observational data, and all the EFO modes were perfectly kept in the observation residual serials of SG data (Lei, 2003).

It is very complex to calculate directly the atmosphere variation effect on the station observation for the correction of air pressure effect. Fortunately a simple method (Farrell, 1972; Niebauer, 1988; Merriam, 1992) was proposed to compute the correction of the air pressure effect, which could be simply expressed as the multiplication of the pressure variation $\Delta P$ and atmospheric gravity admittance
A. Niebauer (1988) and Merriam (1992) showed this method still occupied a high accuracy for the correction of pressure effect. Sun (1997) gained the theoretical atmosphere admittance at Wuhan station $\left(A=-0.3603 \times 10^{-8} \mathrm{~m} \cdot \mathrm{s}^{-2} / \mathrm{hPa}\right)$ with the Green's function computation. Xu et al. (1999) got the experimental atmospheric admittance $\left(A=-0.307 \times 10^{-8} \mathrm{~m} \cdot \mathrm{s}^{-2} / \mathrm{hPa}\right)$ by the analysis of the observation data of SG C032 at Wuhan station. We have tested some different admittance values from $-0.3603 \times 10^{-8} \mathrm{~m} \cdot \mathrm{s}^{-2} / \mathrm{hPa}$ to $-0.307 \times 10^{-8} \mathrm{~m} \cdot \mathrm{s}^{-2} / \mathrm{hPa}$, there was only small difference between these admittances. So it was suitable to adopt the average admittance value $A=-0.326 \times 10^{-8}$ $\mathrm{m} \cdot \mathrm{s}^{-2} / \mathrm{hPa}$ for the correction of pressure effect at Wuhan station. Zürn and Widmer (1995) and Van Camp (1999) considered that the background noise below $1.2 \mathrm{mHz}$ could be effectively decreased after the correction of pressure effect, however, Lei et al. (2002b) showed that the correction of pressure variation produced an effect on the check of EFO signals only below $0.8 \mathrm{mHz}$. As the atmospheric admittance is about $-0.30 \times 10^{-8} \mathrm{~m} \cdot \mathrm{s}^{-2} / \mathrm{hPa}$ in the globe, we applied this value to the correction of pressure effect for other stations. After the correction of gravity tides and pressure effect, the EFO signals have become the main element of the observation residual serials (Lei, 2003).

The SG observation noise is so complicated that there was no analytic description method at present (Van Camp, 1999). The observation noises of SG instruments mainly include the noises of the SG instruments themselves and the local background noise around the SG station. Banka et al. (1998) introduced a method of the noise magnitude of earthquake (abbrev: Mn method) to estimate the observation noises of SG instruments, in which they took the observational residuals spectra in the whole frequency range from $1.67 \mathrm{mHz}$ to $9.9 \mathrm{mHz}$ as the observation noise spectra of SG instruments. However it is clear in fact that there are abundant EFO modes in this frequency range. For this reason, another method was introduced to simulate the observation noises of SG instruments by stacking the observational residuals during the quiet earthquake period (Lei, 2003). The great Sumatra-Andaman earthquake was so huge that there were not quiet recordings for a period after the earthquake, so we stacked the observational noises in the quiet period before this earthquake as the simulation of the observation noise of SG C032.

After applying the fast Fourier transform (FFT) (Ding, 1998) to the observational residuals of SG data, we gained the simulated noise spectrum (SNS) of 
SG instruments. Although SNS are not completely equal to the real noise spectrum of SG instruments during EFO, it also effectively describes the observed noise level of SG instruments during EFO, because the noise level of SG instruments themselves are very stable. We have shown an example of SNS of SG instrument at Wuhan station (Lei et al., 2007). For the accurate estimation on the signal-to-noise ratio (SNR) of every checked EFO mode, we used the average value of SNS in a narrow frequency range covering the mode to present the noise level around it marked as $\mathrm{NL}_{\text {mode }}$ (Lei, 2003). As there were no enough long observation data before the great Sumatra-Andaman earthquake to construct the SNS models of the other four SG stations, we used directly the real observation data during $\mathrm{EFO}$ to build the $\mathrm{NL}_{\text {mode }}$ model for the observed $\mathrm{EFO}$ modes.
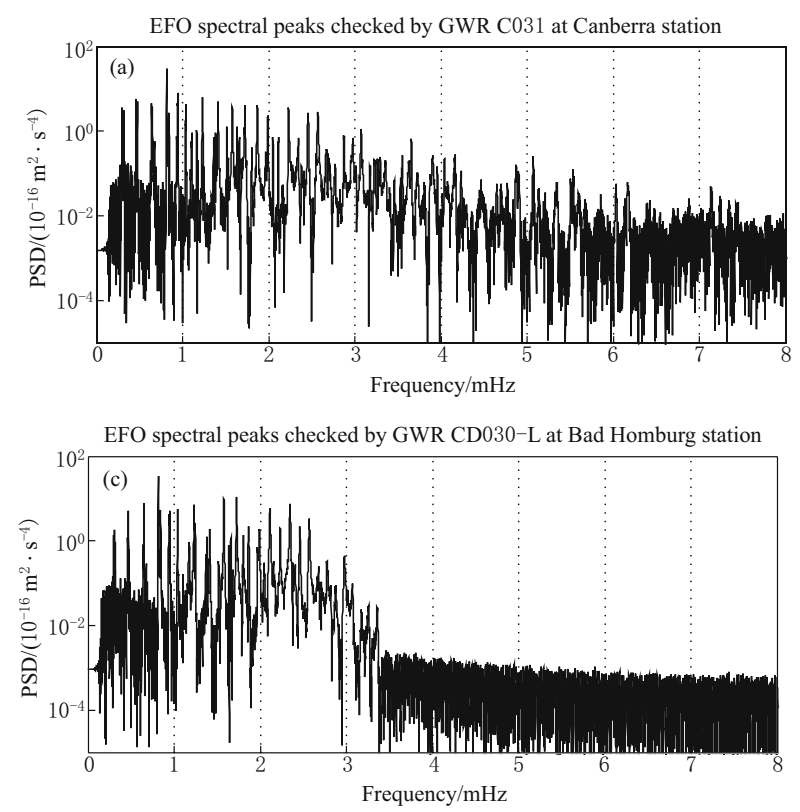

\section{Spectrum analysis and check of the Earth's free oscillation}

The EFO signals are remained as the main element of SG observation residuals after the correction of gravity tides and pressure effect. The EFO spectra were obtained after applying FFT to the observation residuals. The data lengths for the four GGP stations, including Canberra station, Strasbourg station, Bad Homburg station and Wettzell station, were about 394.4 hours and according to the frequency-resolution ratio (FRR) of EFO spectra about $7.04 \times 10^{-7} \mathrm{~Hz}$. We have separately presented the EFO spectra from the four stations mentioned above in Figures 1a-1d. As the low pass filters has been applied to the observation data from Bad
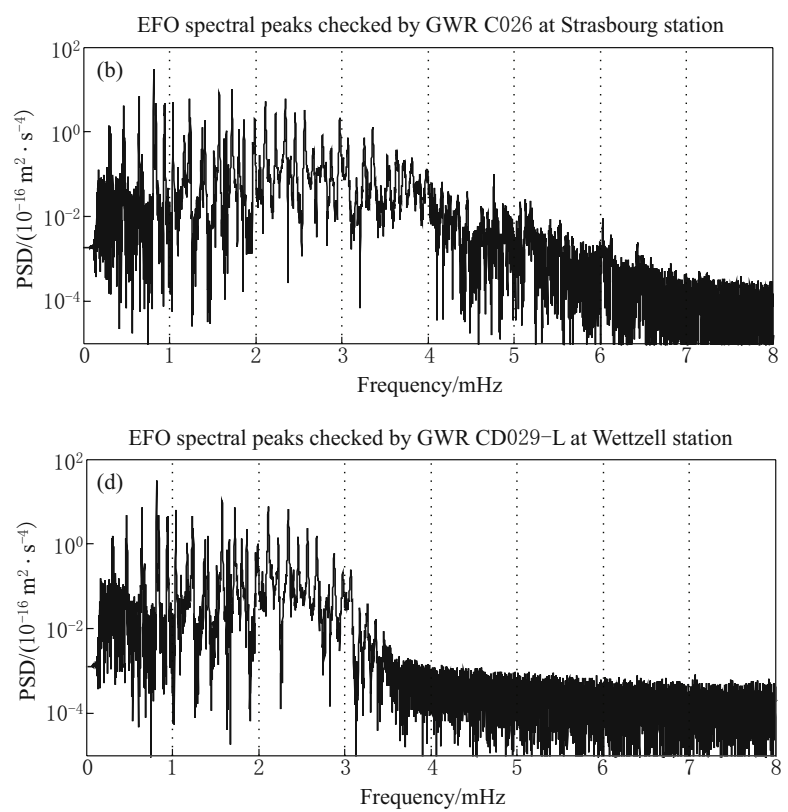

Figure 1 EFO spectral peaks checked by the superconducting gravimeters at four GGP stations. The abscissa is a frequency, the $y$-coordinate is the power spectral density of gravity residuals (PSD). (a) Canberra station; (b) Strasbourg station; (c) Bad Homburg station; (d) Wettzell station.

Homburg station and Wettzell station, there are no EFO mode spectra in the frequency range higher than $3.3 \mathrm{mHz}$. The passage length of observation residuals is about 112.2 hours for Wuhan station and the FRR of EFO spectra is about $2.6 \mu \mathrm{Hz}$ (Lei et al., 2007).

The SNR of every EFO mode was defined as the ratio value between the checked mode peak and the $\mathrm{NL}_{\text {mode }}$ covering the mode. If the SNR of a mode peak was more than 3.0, the checked mode was then considered believable and accepted. We have obtained total of 147 EFO modes including 43 fundamental modes, five radial modes and 99 harmonic modes, five groups of checked mode frequencies as listed in Table 2. The fundamental modes are usually the main element of EFO modes excited by some shallow-focus earthquakes. However, the great Sumatra-Andaman earthquake was so large that it excited not only some fundamental EFO modes but also abundant harmonic modes. It is clear that the excitation of EFO harmonic modes had an uneven distribution in different harmonic degrees. 
Table 2 All EFO modes checked by five GGP stations and comparson with PREM Earth model

\begin{tabular}{|c|c|c|c|c|c|c|c|c|c|}
\hline Mode & $\begin{array}{l}\text { Check1 } \\
/ \mathrm{mHz}\end{array}$ & $\begin{array}{l}\text { Check2 } \\
/ \mathrm{mHz}\end{array}$ & $\begin{array}{l}\text { Check3 } \\
/ \mathrm{mHz}\end{array}$ & $\begin{array}{l}\text { Check4 } \\
/ \mathrm{mHz}\end{array}$ & $\begin{array}{l}\text { Check5 } \\
/ \mathrm{mHz}\end{array}$ & $\begin{array}{c}\text { PREM } \\
/ \mathrm{mHz}\end{array}$ & $\begin{array}{l}\text { Check } \\
/ \mathrm{mHz}\end{array}$ & $\begin{array}{l}\mathrm{DEM} \\
/ \mathrm{mHz}\end{array}$ & RDEM \\
\hline \multicolumn{10}{|c|}{ Radial modes } \\
\hline${ }_{0} \mathrm{~S}_{0}$ & 0.81433 & 0.81433 & 0.81433 & 0.81433 & 0.8145 & 0.81431 & 0.814342 & 0.000032 & $0.004 \%$ \\
\hline${ }_{1} \mathrm{~S}_{0}$ & 1.63148 & 1.63148 & 1.63148 & 1.63148 & 1.6315 & 1.63134 & 1.631481 & 0.000141 & $0.009 \%$ \\
\hline${ }_{3} \mathrm{~S}_{0}$ & 3.27421 & 3.2728 & & & & 3.27118 & 3.273505 & 0.002325 & $0.071 \%$ \\
\hline${ }_{4} \mathrm{~S}_{0}$ & & 4.10755 & & & 4.1097 & 4.10576 & 4.108037 & 0.002277 & $0.055 \%$ \\
\hline${ }_{8} \mathrm{~S}_{0}$ & 7.4268 & & & & & 7.42413 & 7.426800 & 0.002670 & $0.036 \%$ \\
\hline \multicolumn{10}{|c|}{ Fundamental modes } \\
\hline${ }_{0} \mathrm{~S}_{2}$ & 0.309333 & 0.308981 & 0.308981 & 0.308981 & 0.3095 & 0.30928 & 0.309098 & -0.000182 & $-0.059 \%$ \\
\hline${ }_{0} \mathrm{~S}_{3}$ & 0.468398 & 0.468705 & 0.46875 & 0.46875 & 0.4687 & 0.46856 & 0.468654 & 0.000094 & $0.020 \%$ \\
\hline${ }_{0} \mathrm{~S}_{4}$ & 0.646115 & 0.646819 & 0.646819 & 0.646819 & 0.6474 & 0.64707 & 0.646695 & -0.000375 & $-0.058 \%$ \\
\hline${ }_{0} \mathrm{~S}_{5}$ & 0.839316 & 0.840372 & 0.840372 & 0.840372 & 0.8393 & 0.84042 & 0.840053 & -0.000367 & $-0.044 \%$ \\
\hline${ }_{0} \mathrm{~S}_{6}$ & 1.03956 & 1.03956 & 1.03956 & 1.03956 & 1.0386 & 1.03822 & 1.039495 & 0.001275 & $0.123 \%$ \\
\hline${ }_{0} \mathrm{~S}_{7}$ & 1.2324 & 1.2324 & 1.2324 & 1.2317 & 1.2304 & 1.23179 & 1.232101 & 0.000311 & $0.025 \%$ \\
\hline${ }_{0} \mathrm{~S}_{8}$ & 1.41329 & 1.41399 & 1.41399 & 1.41329 & 1.4112 & 1.41351 & 1.413474 & -0.000036 & $-0.003 \%$ \\
\hline${ }_{0} \mathrm{~S}_{9}$ & 1.57728 & 1.57728 & 1.57728 & 1.57658 & 1.577 & 1.57828 & 1.577098 & -0.001182 & $-0.075 \%$ \\
\hline${ }_{0} \mathrm{~S}_{10}$ & 1.72368 & & 1.72368 & & 1.7256 & 1.72647 & 1.723925 & -0.002545 & $-0.147 \%$ \\
\hline${ }_{0} \mathrm{~S}_{11}$ & & 1.86303 & 1.86374 & 1.86303 & 1.8642 & 1.86242 & 1.863350 & 0.000930 & $0.050 \%$ \\
\hline${ }_{0} \mathrm{~S}_{12}$ & 1.99113 & 1.98902 & & 1.99324 & 1.9905 & 1.99037 & 1.991074 & 0.000704 & $0.035 \%$ \\
\hline${ }_{0} \mathrm{~S}_{13}$ & 2.11289 & 2.11289 & 2.11219 & 2.11289 & 2.1143 & 2.11294 & 2.112823 & -0.000117 & $-0.006 \%$ \\
\hline${ }_{0} \mathrm{~S}_{14}$ & 2.23043 & 2.23255 & 2.23114 & 2.22903 & 2.2331 & 2.2314 & 2.230945 & -0.000455 & $-0.020 \%$ \\
\hline${ }_{0} \mathrm{~S}_{15}$ & 2.34516 & 2.34586 & 2.34586 & & 2.347 & 2.34638 & 2.345749 & -0.000631 & $-0.027 \%$ \\
\hline${ }_{0} \mathrm{~S}_{16}$ & 2.45707 & 2.45918 & 2.45918 & 2.45847 & 2.4609 & 2.45822 & 2.458640 & 0.000420 & $0.017 \%$ \\
\hline${ }_{0} \mathrm{~S}_{17}$ & 2.57038 & 2.56827 & 2.56757 & 2.56827 & 2.5698 & 2.56712 & 2.568703 & 0.001583 & $0.062 \%$ \\
\hline${ }_{0} \mathrm{~S}_{18}$ & 2.67314 & 2.67314 & 2.67666 & 2.67455 & 2.6763 & 2.6733 & 2.674504 & 0.001204 & $0.045 \%$ \\
\hline${ }_{0} \mathrm{~S}_{19}$ & 2.77309 & 2.77449 & 2.77309 & & 2.7778 & 2.77698 & 2.773934 & -0.003046 & $-0.110 \%$ \\
\hline${ }_{0} \mathrm{~S}_{20}$ & 2.87584 & & 2.87866 & 2.87866 & 2.8793 & 2.87837 & 2.877860 & -0.000510 & $-0.018 \%$ \\
\hline${ }_{0} \mathrm{~S}_{21}$ & 2.97649 & 2.97649 & & 2.9786 & 2.9783 & 2.97772 & 2.977292 & -0.000428 & $-0.014 \%$ \\
\hline${ }_{0} \mathrm{~S}_{22}$ & 3.07362 & 3.07151 & 3.07151 & 3.07151 & 3.0749 & 3.07527 & 3.072233 & -0.003037 & $-0.099 \%$ \\
\hline${ }_{0} \mathrm{~S}_{23}$ & 3.17638 & & 3.17145 & 3.17286 & 3.1714 & 3.17126 & 3.173371 & 0.002111 & $0.067 \%$ \\
\hline${ }_{0} \mathrm{~S}_{24}$ & 3.26365 & 3.26295 & & 3.26436 & 3.2655 & 3.26589 & 3.263817 & -0.002073 & $-0.063 \%$ \\
\hline${ }_{0} \mathrm{~S}_{25}$ & 3.35586 & 3.35797 & 3.35726 & 3.35797 & 3.3621 & 3.35938 & 3.357595 & -0.001785 & $-0.053 \%$ \\
\hline${ }_{0} \mathrm{~S}_{26}$ & 3.4558 & 3.45087 & & & 3.4561 & 3.45191 & 3.453688 & 0.001778 & $0.052 \%$ \\
\hline${ }_{0} \mathrm{~S}_{27}$ & & & & & 3.5453 & 3.54365 & 3.545300 & 0.001650 & $0.047 \%$ \\
\hline${ }_{0} \mathrm{~S}_{28}$ & 3.62894 & 3.63316 & & & 3.6344 & 3.63476 & 3.631478 & -0.003282 & $-0.090 \%$ \\
\hline${ }_{0} \mathrm{~S}_{29}$ & 3.72537 & 3.72396 & & & 3.7235 & 3.72534 & 3.724516 & -0.000824 & $-0.022 \%$ \\
\hline${ }_{0} \mathrm{~S}_{30}$ & & 3.81334 & & & 3.8176 & 3.81552 & 3.814305 & -0.001215 & $-0.032 \%$ \\
\hline${ }_{0} \mathrm{~S}_{31}$ & 3.90343 & 3.90273 & & & 3.9067 & 3.9054 & 3.903542 & -0.001858 & $-0.048 \%$ \\
\hline${ }_{0} \mathrm{~S}_{32}$ & & & & & 3.9983 & 3.99504 & 3.998300 & 0.003260 & $0.082 \%$ \\
\hline${ }_{0} \mathrm{~S}_{33}$ & 4.07869 & & & & & 4.08452 & 4.078690 & -0.005830 & $-0.143 \%$ \\
\hline${ }_{0} \mathrm{~S}_{34}$ & 4.17019 & & & & 4.1716 & 4.1739 & 4.170509 & -0.003391 & $-0.081 \%$ \\
\hline${ }_{0} \mathrm{~S}_{35}$ & & 4.26168 & & & 4.2583 & 4.26323 & 4.260915 & -0.002315 & $-0.054 \%$ \\
\hline${ }_{0} \mathrm{~S}_{36}$ & & & & & 4.3548 & 4.35253 & 4.354800 & 0.002270 & $0.052 \%$ \\
\hline${ }_{0} \mathrm{~S}_{37}$ & & & & & 4.4415 & 4.44184 & 4.441500 & -0.000340 & $-0.008 \%$ \\
\hline${ }_{0} \mathrm{~S}_{38}$ & & & & & 4.5281 & 4.5312 & 4.528100 & -0.003100 & $-0.068 \%$ \\
\hline${ }_{0} \mathrm{~S}_{39}$ & & & & & 4.6222 & 4.62061 & 4.622200 & 0.001590 & $0.034 \%$ \\
\hline${ }_{0} \mathrm{~S}_{40}$ & & & & & 4.7113 & 4.7101 & 4.711300 & 0.001200 & $0.025 \%$ \\
\hline${ }_{0} \mathrm{~S}_{41}$ & & & & & 4.793 & 4.79967 & 4.793000 & -0.006670 & $-0.139 \%$ \\
\hline${ }_{0} \mathrm{~S}_{42}$ & 4.88739 & & & & 4.8896 & 4.88934 & 4.887890 & -0.001450 & $-0.030 \%$ \\
\hline${ }_{0} \mathrm{~S}_{44}$ & 5.07038 & & & & 5.0654 & 5.06901 & 5.069252 & 0.000242 & $0.005 \%$ \\
\hline${ }_{0} \mathrm{~S}_{47}$ & 5.34136 & & & & & 5.33935 & 5.341360 & 0.002010 & $0.038 \%$ \\
\hline
\end{tabular}


Continued from Table 2

\begin{tabular}{|c|c|c|c|c|c|c|c|c|c|}
\hline Mode & $\begin{array}{l}\text { Check1 } \\
/ \mathrm{mHz}\end{array}$ & $\begin{array}{l}\text { Check2 } \\
/ \mathrm{mHz}\end{array}$ & $\begin{array}{c}\text { Check3 } \\
/ \mathrm{mHz}\end{array}$ & $\begin{array}{l}\text { Check4 } \\
/ \mathrm{mHz}\end{array}$ & $\begin{array}{l}\text { Check5 } \\
/ \mathrm{mHz}\end{array}$ & $\begin{array}{c}\text { PREM } \\
/ \mathrm{mHz}\end{array}$ & $\begin{array}{l}\text { Check } \\
/ \mathrm{mHz}\end{array}$ & $\begin{array}{l}\mathrm{DEM} \\
/ \mathrm{mHz}\end{array}$ & RDEM \\
\hline \multicolumn{10}{|c|}{ 1-degree harmonic modes } \\
\hline${ }_{1} \mathrm{~S}_{2}$ & & 0.677787 & 0.677787 & 0.677787 & 0.6808 & 0.67985 & 0.678055 & -0.001795 & $-0.264 \%$ \\
\hline${ }_{1} \mathrm{~S}_{4}$ & 1.16848 & 1.17188 & 1.16976 & 1.17117 & 1.1747 & 1.17285 & 1.170621 & -0.002229 & $-0.190 \%$ \\
\hline${ }_{1} \mathrm{~S}_{5}$ & 1.36895 & 1.37035 & 1.37106 & 1.36895 & & 1.37027 & 1.371919 & 0.001649 & $0.120 \%$ \\
\hline${ }_{1} \mathrm{~S}_{6}$ & 1.52872 & 1.52168 & 1.52168 & 1.52731 & 1.5251 & 1.52204 & 1.524865 & 0.002825 & $0.186 \%$ \\
\hline${ }_{1} \mathrm{~S}_{7}$ & 1.65892 & 1.65892 & 1.65892 & 1.65963 & 1.6538 & 1.65551 & 1.658736 & 0.003226 & $0.195 \%$ \\
\hline${ }_{1} \mathrm{~S}_{8}$ & 1.79828 & 1.79969 & 1.80251 & 1.79617 & 1.8023 & 1.7993 & 1.799376 & 0.000076 & $0.004 \%$ \\
\hline${ }_{1} \mathrm{~S}_{9}$ & 1.9672 & 1.96791 & 1.9672 & 1.9672 & 1.9657 & 1.96374 & 1.967263 & 0.003523 & $0.179 \%$ \\
\hline${ }_{1} \mathrm{~S}_{10}$ & & 2.15231 & 2.1516 & 2.1516 & & 2.14842 & 2.151837 & 0.003417 & $0.159 \%$ \\
\hline${ }_{1} \mathrm{~S}_{11}$ & & & & 2.34586 & & 2.34754 & 2.345860 & -0.001680 & $-0.072 \%$ \\
\hline${ }_{1} \mathrm{~S}_{14}$ & & & 2.97649 & 2.97508 & & 2.97579 & 2.975785 & -0.000005 & $0.000 \%$ \\
\hline${ }_{1} \mathrm{~S}_{15}$ & & 3.17005 & & & & 3.17055 & 3.170050 & -0.000500 & $-0.016 \%$ \\
\hline${ }_{1} \mathrm{~S}_{18}$ & & & & & 3.6418 & 3.64494 & 3.641800 & -0.003140 & $-0.086 \%$ \\
\hline${ }_{1} \mathrm{~S}_{22}$ & & & & & 4.231 & 4.2344 & 4.231000 & -0.003400 & $-0.080 \%$ \\
\hline${ }_{1} \mathrm{~S}_{29}$ & & & & & 5.2387 & 5.23929 & 5.238700 & -0.000590 & $-0.011 \%$ \\
\hline \multicolumn{10}{|c|}{ 2-degree harmonic modes } \\
\hline${ }_{2} \mathrm{~S}_{2}$ & 0.932221 & 0.936092 & 0.936092 & 0.936092 & & 0.93785 & 0.935124 & -0.002726 & $-0.291 \%$ \\
\hline${ }_{2} \mathrm{~S}_{3}$ & 1.24367 & 1.24437 & 1.24437 & 1.24437 & 1.2453 & 1.24219 & 1.244270 & 0.002080 & $0.167 \%$ \\
\hline${ }_{2} \mathrm{~S}_{4}$ & 1.38091 & 1.38091 & 1.38091 & & 1.3765 & 1.3792 & 1.380518 & 0.001318 & $0.095 \%$ \\
\hline${ }_{2} \mathrm{~S}_{5}$ & 1.51323 & & 1.51605 & 1.51605 & 1.5127 & 1.51493 & 1.514896 & -0.000034 & $-0.002 \%$ \\
\hline${ }_{2} \mathrm{~S}_{6}$ & 1.68285 & 1.68074 & 1.68074 & & 1.6835 & 1.68084 & 1.681626 & 0.000786 & $0.047 \%$ \\
\hline${ }_{2} \mathrm{~S}_{7}$ & 1.86726 & & & & & 1.86496 & 1.867260 & 0.002300 & $0.123 \%$ \\
\hline${ }_{2} \mathrm{~S}_{10}$ & 2.40428 & 2.40358 & 2.40498 & & & 2.40293 & 2.404280 & 0.001350 & $0.056 \%$ \\
\hline${ }_{2} \mathrm{~S}_{16}$ & & & & & 3.4438 & 3.44346 & 3.443800 & 0.000340 & $0.010 \%$ \\
\hline${ }_{2} \mathrm{~S}_{18}$ & 3.87247 & 3.87528 & & & & 3.87444 & 3.873875 & -0.000565 & $-0.015 \%$ \\
\hline \multicolumn{10}{|c|}{ 3-degree harmonic modes } \\
\hline${ }_{3} \mathrm{~S}_{1}$ & 0.944538 & 0.944538 & 0.944538 & 0.944538 & 0.9457 & 0.94395 & 0.944617 & 0.000667 & $0.071 \%$ \\
\hline${ }_{3} \mathrm{~S}_{2}$ & 1.09938 & 1.10008 & 1.10008 & 1.10008 & 1.1029 & 1.10621 & 1.100109 & -0.006101 & $-0.552 \%$ \\
\hline${ }_{3} \mathrm{~S}_{6}$ & 2.55068 & & & & & 2.54964 & 2.550680 & 0.001040 & $0.041 \%$ \\
\hline${ }_{3} \mathrm{~S}_{7}$ & 2.69074 & & & & & 2.68633 & 2.690740 & 0.004410 & $0.164 \%$ \\
\hline${ }_{3} \mathrm{~S}_{8}$ & & & & 2.81954 & 2.8199 & 2.81964 & 2.819622 & -0.000018 & $-0.001 \%$ \\
\hline${ }_{3} \mathrm{~S}_{10}$ & 3.08699 & & & & & 3.08479 & 3.086990 & 0.002200 & $0.071 \%$ \\
\hline${ }_{3} \mathrm{~S}_{13}$ & & 3.50366 & & & & 3.50744 & 3.503660 & -0.003780 & $-0.108 \%$ \\
\hline${ }_{3} \mathrm{~S}_{14}$ & 3.65639 & 3.65639 & & & & 3.65711 & 3.656390 & -0.000720 & $-0.020 \%$ \\
\hline${ }_{3} \mathrm{~S}_{15}$ & 3.80771 & & & & 3.8126 & 3.81048 & 3.808817 & -0.001663 & $-0.044 \%$ \\
\hline${ }_{3} \mathrm{~S}_{19}$ & 4.4489 & & & & & 4.44756 & 4.448900 & 0.001340 & $0.030 \%$ \\
\hline${ }_{3} \mathrm{~S}_{21}$ & & & & & 4.7732 & 4.77264 & 4.773200 & 0.000560 & $0.012 \%$ \\
\hline \multicolumn{10}{|c|}{ 4-degree harmonic modes } \\
\hline${ }_{4} \mathrm{~S}_{1}$ & & & 1.40836 & & & 1.41264 & 1.408360 & -0.004280 & $-0.303 \%$ \\
\hline${ }_{4} \mathrm{~S}_{2}$ & & 1.72368 & & 1.72368 & 1.7206 & 1.7223 & 1.723287 & 0.000987 & $0.057 \%$ \\
\hline${ }_{4} \mathrm{~S}_{3}$ & 2.04885 & 2.04392 & 2.04462 & 2.04332 & & 2.04896 & 2.045178 & -0.003783 & $-0.185 \%$ \\
\hline${ }_{4} \mathrm{~S}_{7}$ & & 3.01591 & 3.01661 & 3.01661 & & 3.01363 & 3.016377 & 0.002747 & $0.091 \%$ \\
\hline${ }_{4} \mathrm{~S}_{9}$ & & 3.71129 & & & 3.7087 & 3.70876 & 3.710704 & 0.001944 & $0.052 \%$ \\
\hline${ }_{4} \mathrm{~S}_{10}$ & & 3.86684 & & & & 3.86458 & 3.866840 & 0.002260 & $0.058 \%$ \\
\hline${ }_{4} \mathrm{~S}_{12}$ & & 4.15189 & & & & 4.15297 & 4.151890 & -0.001080 & $-0.026 \%$ \\
\hline${ }_{4} \mathrm{~S}_{19}$ & & & & & 5.2065 & 5.20651 & 5.206500 & -0.000010 & $0.000 \%$ \\
\hline \multicolumn{10}{|c|}{ 5-degree harmonic modes } \\
\hline${ }_{5} \mathrm{~S}_{1}$ & 1.71734 & & & & & 1.71379 & 1.717340 & 0.003550 & $0.207 \%$ \\
\hline${ }_{5} \mathrm{~S}_{3}$ & & 2.1692 & 2.1692 & 2.1692 & & 2.16966 & 2.169200 & -0.000460 & $-0.021 \%$ \\
\hline
\end{tabular}


Continued from Table 2

\begin{tabular}{|c|c|c|c|c|c|c|c|c|c|}
\hline Mode & $\begin{array}{c}\text { Check1 } \\
\text { /mHz }\end{array}$ & $\begin{array}{l}\text { Check2 } \\
/ \mathrm{mHz}\end{array}$ & $\begin{array}{c}\text { Check3 } \\
/ \mathrm{mHz}\end{array}$ & $\begin{array}{l}\text { Check4 } \\
/ \mathrm{mHz}\end{array}$ & $\begin{array}{c}\text { Check5 } \\
/ \mathrm{mHz}\end{array}$ & $\begin{array}{c}\text { PREM } \\
/ \mathrm{mHz}\end{array}$ & $\begin{array}{l}\text { Check } \\
/ \mathrm{mHz}\end{array}$ & $\begin{array}{l}\mathrm{DEM} \\
/ \mathrm{mHz}\end{array}$ & RDEM \\
\hline${ }_{5} \mathrm{~S}_{4}$ & 2.37965 & 2.38035 & 2.38035 & 2.38035 & 2.378 & 2.37952 & 2.380027 & 0.000507 & $0.021 \%$ \\
\hline${ }_{5} \mathrm{~S}_{5}$ & 2.702 & & 2.70763 & 2.70763 & 2.7035 & 2.70336 & 2.705553 & 0.002193 & $0.081 \%$ \\
\hline${ }_{5} \mathrm{~S}_{6}$ & 3.01028 & & & & & 3.01069 & 3.010280 & -0.000410 & $-0.014 \%$ \\
\hline${ }_{5} \mathrm{~S}_{7}$ & 3.29603 & 3.29603 & & & 3.2952 & 3.29076 & 3.295924 & 0.005164 & $0.157 \%$ \\
\hline${ }_{5} \mathrm{~S}_{8}$ & 3.53111 & 3.52759 & & & 3.5279 & 3.52565 & 3.529165 & 0.003515 & $0.100 \%$ \\
\hline${ }_{5} \mathrm{~S}_{10}$ & & 4.15541 & & & & 4.157 & 4.155410 & -0.001590 & $-0.038 \%$ \\
\hline${ }_{5} \mathrm{~S}_{11}$ & & 4.45524 & & & 4.4588 & 4.45655 & 4.456046 & -0.000504 & $-0.011 \%$ \\
\hline${ }_{5} \mathrm{~S}_{13}$ & & & & & 4.9218 & 4.9244 & 4.921800 & -0.002600 & $-0.053 \%$ \\
\hline${ }_{5} \mathrm{~S}_{14}$ & 5.13654 & 5.13443 & & & 5.1322 & 5.13681 & 5.135066 & -0.001744 & $-0.034 \%$ \\
\hline${ }_{5} \mathrm{~S}_{15}$ & & & & & 5.3303 & 5.33011 & 5.330300 & 0.000190 & $0.004 \%$ \\
\hline${ }_{5} \mathrm{~S}_{16}$ & 5.50535 & & & & & 5.50696 & 5.505350 & -0.001610 & $-0.029 \%$ \\
\hline${ }_{5} \mathrm{~S}_{17}$ & 5.66864 & & & & & 5.67369 & 5.668640 & -0.005050 & $-0.089 \%$ \\
\hline \multicolumn{10}{|c|}{ 6-degree harmonic modes } \\
\hline${ }_{6} \mathrm{~S}_{3}$ & & & 2.82095 & & 2.8248 & 2.82172 & 2.821822 & 0.000102 & $0.004 \%$ \\
\hline${ }_{6} \mathrm{~S}_{4}$ & & 3.08981 & & & & 3.09211 & 3.089810 & -0.002300 & $-0.074 \%$ \\
\hline${ }_{6} \mathrm{~S}_{6}$ & 3.40442 & & & & & 3.40386 & 3.404420 & 0.000560 & $0.016 \%$ \\
\hline${ }_{6} \mathrm{~S}_{7}$ & 3.55152 & 3.55082 & & & 3.5502 & 3.5526 & 3.551046 & -0.001554 & $-0.044 \%$ \\
\hline${ }_{6} \mathrm{~S}_{10}$ & & 4.20538 & & & & 4.21076 & 4.205380 & -0.005380 & $-0.128 \%$ \\
\hline${ }_{6} \mathrm{~S}_{13}$ & 5.23438 & & & & & 5.23388 & 5.234380 & 0.000500 & $0.010 \%$ \\
\hline${ }_{6} \mathrm{~S}_{15}$ & 5.59755 & & & & & 5.60251 & 5.597550 & -0.004960 & $-0.089 \%$ \\
\hline \multicolumn{10}{|c|}{ 7-degree harmonic modes } \\
\hline${ }_{7} \mathrm{~S}_{5}$ & & & & & 3.6616 & 3.65975 & 3.661600 & 0.001850 & $0.051 \%$ \\
\hline${ }_{7} \mathrm{~S}_{6}$ & 3.95763 & & & & 3.9612 & 3.95872 & 3.958438 & -0.000282 & $-0.007 \%$ \\
\hline${ }_{7} \mathrm{~S}_{7}$ & 4.23635 & 4.23775 & & & 4.236 & 4.23785 & 4.236916 & -0.000934 & $-0.022 \%$ \\
\hline${ }_{7} \mathrm{~S}_{8}$ & & & & & 4.4489 & 4.45259 & 4.448900 & -0.003690 & $-0.083 \%$ \\
\hline${ }_{7} \mathrm{~S}_{10}$ & & & & & 4.7584 & 4.76776 & 4.758400 & -0.009360 & $-0.196 \%$ \\
\hline${ }_{7} \mathrm{~S}_{11}$ & & & & & 4.9143 & 4.91694 & 4.914300 & -0.002640 & $-0.054 \%$ \\
\hline${ }_{7} \mathrm{~S}_{13}$ & & & & & 5.2808 & 5.28815 & 5.280800 & -0.007350 & $-0.139 \%$ \\
\hline${ }_{7} \mathrm{~S}_{18}$ & 6.75042 & & & & & 6.76628 & 6.750420 & -0.015860 & $-0.234 \%$ \\
\hline \multicolumn{10}{|c|}{ 8-degree harmonic modes } \\
\hline${ }_{8} \mathrm{~S}_{1}$ & 2.87233 & & 2.87021 & & 2.8719 & 2.87336 & 2.871350 & -0.002010 & $-0.070 \%$ \\
\hline${ }_{8} \mathrm{~S}_{3}$ & 3.28477 & & & & & 3.28364 & 3.284770 & 0.001130 & $0.034 \%$ \\
\hline${ }_{8} \mathrm{~S}_{5}$ & 4.16174 & 4.16807 & & & 4.1667 & 4.1662 & 4.165134 & -0.001066 & $-0.026 \%$ \\
\hline${ }_{8} \mathrm{~S}_{6}$ & & & & & 4.4316 & 4.43524 & 4.431600 & -0.003640 & $-0.082 \%$ \\
\hline${ }_{8} \mathrm{~S}_{7}$ & 4.64809 & & & & & 4.65046 & 4.648090 & -0.002370 & $-0.051 \%$ \\
\hline${ }_{8} \mathrm{~S}_{8}$ & 4.90287 & & & & & 4.90807 & 4.902870 & -0.005200 & $-0.106 \%$ \\
\hline${ }_{8} \mathrm{~S}_{9}$ & 5.21185 & 5.20974 & & & & 5.21187 & 5.210795 & -0.001075 & $-0.021 \%$ \\
\hline \multicolumn{10}{|c|}{ 9-degree harmonic modes } \\
\hline${ }_{9} \mathrm{~S}_{2}$ & 3.22987 & 3.23057 & & & 3.2284 & 3.23175 & 3.229988 & -0.001762 & $-0.055 \%$ \\
\hline${ }_{9} \mathrm{~S}_{4}$ & 3.87669 & & & & 3.8721 & 3.87795 & 3.875651 & -0.002299 & $-0.059 \%$ \\
\hline${ }_{9} \mathrm{~S}_{7}$ & 4.87261 & & & & & 4.87264 & 4.872610 & -0.000030 & $-0.001 \%$ \\
\hline \multicolumn{10}{|c|}{ More than 10-degree harmonic modes } \\
\hline${ }_{10} \mathrm{~S}_{2}$ & 4.04068 & 4.04139 & & & 4.0355 & 4.03233 & 4.040328 & 0.007998 & $0.198 \%$ \\
\hline${ }_{10} \mathrm{~S}_{5}$ & 4.4672 & & & & 4.4687 & 4.46795 & 4.467540 & -0.000410 & $-0.009 \%$ \\
\hline${ }_{11} \mathrm{~S}_{3}$ & 4.46439 & 4.45946 & & & & 4.46242 & 4.461925 & -0.000495 & $-0.011 \%$ \\
\hline${ }_{11} \mathrm{~S}_{4}$ & & 4.76633 & & & & 4.76686 & 4.766330 & -0.000530 & $-0.011 \%$ \\
\hline${ }_{11} \mathrm{~S}_{5}$ & & 5.07531 & & & 5.0728 & 5.07441 & 5.074742 & 0.000332 & $0.007 \%$ \\
\hline${ }_{12} \mathrm{~S}_{1}$ & & & & & 4.3078 & 4.30034 & 4.307800 & 0.007460 & $0.173 \%$ \\
\hline${ }_{12} \mathrm{~S}_{2}$ & & 4.34192 & & & & 4.33016 & 4.341920 & 0.011760 & $0.272 \%$ \\
\hline
\end{tabular}




\begin{tabular}{|c|c|c|c|c|c|c|c|c|c|}
\hline & & & & & & & & \multicolumn{2}{|c|}{ Continued from Table } \\
\hline Mode & $\begin{array}{c}\text { Check1 } \\
/ \mathrm{mHz}\end{array}$ & $\begin{array}{c}\text { Check2 } \\
/ \mathrm{mHz}\end{array}$ & $\begin{array}{c}\text { Check3 } \\
/ \mathrm{mHz}\end{array}$ & $\begin{array}{c}\text { Check4 } \\
/ \mathrm{mHz}\end{array}$ & $\begin{array}{c}\text { Check5 } \\
/ \mathrm{mHz}\end{array}$ & $\begin{array}{c}\text { PREM } \\
/ \mathrm{mHz}\end{array}$ & $\begin{array}{l}\text { Check } \\
/ \mathrm{mHz}\end{array}$ & $\begin{array}{l}\mathrm{DEM} \\
/ \mathrm{mHz}\end{array}$ & RDEM \\
\hline${ }_{12} \mathrm{~S}_{5}$ & & 5.52928 & & & & 5.52766 & 5.529280 & 0.001620 & $0.029 \%$ \\
\hline${ }_{12} \mathrm{~S}_{7}$ & 5.85515 & & & & & 5.85588 & 5.855150 & -0.000730 & $-0.012 \%$ \\
\hline${ }_{12} \mathrm{~S}_{8}$ & 6.13739 & & & & & 6.13717 & 6.137390 & 0.000220 & $0.004 \%$ \\
\hline${ }_{13} \mathrm{~S}_{2}$ & 4.83812 & & & & 4.8475 & 4.84526 & 4.840244 & -0.005016 & $-0.104 \%$ \\
\hline${ }_{13} \mathrm{~S}_{3}$ & 5.19566 & 5.19074 & & & 5.1941 & 5.19382 & 5.193315 & -0.000505 & $-0.010 \%$ \\
\hline${ }_{13} \mathrm{~S}_{6}$ & 6.16484 & & & & & 6.16119 & 6.164840 & 0.003650 & $0.059 \%$ \\
\hline${ }_{15} \mathrm{~S}_{3}$ & 6.03533 & 6.03392 & & & & 6.03522 & 6.034625 & -0.000595 & $-0.010 \%$ \\
\hline${ }_{15} \mathrm{~S}_{5}$ & 6.47382 & & & & & 6.47531 & 6.473820 & -0.001490 & $-0.023 \%$ \\
\hline${ }_{17} \mathrm{~S}_{2}$ & 6.39147 & & & & & 6.39521 & 6.391470 & -0.003740 & $-0.058 \%$ \\
\hline${ }_{18} \mathrm{~S}_{4}$ & 7.2431 & & & & & 7.241 & 7.243100 & 0.002100 & $0.029 \%$ \\
\hline${ }_{19} \mathrm{~S}_{1}$ & 6.42314 & & & & & 6.42744 & 6.423140 & -0.004300 & $-0.067 \%$ \\
\hline
\end{tabular}

Notes: The serials of check1, check2, check3, check4 and check5 are the observed values of EFO modes checked separately by Canberra station, Strasbourg station, Bad Homburg station, Wettzell station and Wuhan station. Among the five serials of EFO modes, the group of check5 serials refer to our former paper (Lei et al., 2007), the other check serials are all the new observation results provided in this paper. The signal-to-noise ratios (SNR) of all EFO modes are more than 3. The frequency-resolution ratios (FRR) of check1, check2, check3 and check4 serials are approximately equal to $7.04 \times 10^{-7} \mathrm{~Hz}$ and that of check5 serials is about $2.6 \times 10^{-7} \mathrm{~Hz}$. The serials of PREM are the frequency values based on the PREM Earth model. Check serials are the weighted average values of all observations provided by the five SG stations, and the weighted parameters are according to their FRR. The serials of DEM are the frequency differences between the check serials and the PREM serials. The serials of RDEM are the ratio values of the DEM serials to the PREM serials.

The great Sumatra-Andaman earthquake fully generated the 1-degree (14 modes), 3-degree (11 modes) and 5 -degree (14 modes) harmonic modes. To determine the final check results of EFO modes, the weighted average value of observed frequencies has been calculated for every checked EFO mode and the weighted parameters are got according to the reciprocal values of the FRR of every observation data serials.

All check values of EFO modes are listed in Table 2. In order to compare the experimental EFO modes with the theoretical ones (Dziewonski and Anderson, 1981), we list the theoretical predictions of EFO modes in Table 2 based on the PREM model. The deviation of EFO modes (DEM) are defined as the frequency differences between the checked modes and the theoretical modes. The relative deviation of EFO modes (RDEM) are defined as the ratios of the DEM values to the theoretical mode frequencies. Two group serials of checked modes' DEM and RDEM are also listed in Table 2. As the theoretical frequencies of EFO modes are directly related to the geophysical parameters of the PREM Earth model, the RDEM serials of experimental EFO modes can provide some valuable information on the Earth's deep structure, which helps to improve the known Earth model. However if the DEM value of an EFO mode was not more than a multiple of FRR $7.04 \times 10^{-7} \mathrm{~Hz}$, it would be unsuitable to discuss the Earth's deep structure with the RDEM value of the mode. On the other hand, the experimental DEM and RDEM serials are also taken as the evaluating factors of the observation quality. Our observation results provide a new set of high quality and fairly complete data serials of experimental EFO modes with SG instruments, which is helpful to improve the study of the Earth's deep structure.

\section{Discussions on the structure of the Earth's inner core}

The Earth's inner core with a radius of $1221.5 \mathrm{~km}$ is an important deep structure in the Earth (Dziewonski and Anderson, 1981; Tromp, 1993). Some geophysical phenomena are directly related to the structure of the Earth's inner core. Poupinet et al. (1983) found the abnormal travel times of PKIKP body waves passing through the Earth's inner core, and Masters and Gilbert (1981) noticed the anomalous spectral splitting of EFO. To explain these abnormal geophysical phenomena, Morelli et al. (1986) and Woodhouse et al. (1986) introduced the conception of the inner core anisotropy and considered that the inner core anisotropy was axisymmetric. Birch (1964) measured the density and sound speed of different elements with the experiments and manifested that the iron was possibly the only one of main elements whose density under the pressure of the inner core was in agreement with the density of the inner core. Some mineralogy investigations displayed 
that the hexagonal close packed (hcp) iron ( $\varepsilon$-phase) was the most likely phase on the conditions of the inner core, and the directional distributions of $\varepsilon$-phase iron caused the anisotropy of the inner core (Anderson, 1995; Stixrude and Cohen, 1995). Creager (1992) and Shearer (1994) discovered that in the inner core $\mathrm{P}$ wave velocity in the north-south direction was faster than that in the equatorial direction by about $3 \%$. Laske and Masters (1999) analyzed the differential rotation of the inner core with EFO mode splittings. Ishii et al. (2002) and Beghein and Trampert (2003) discussed carefully the anisotropy of the inner core.

But there were still some arguments on the structure of the Earth's inner core between the $\mathrm{P}$ wave results and EFO observation. Shearer and Masters (1990) and Song and Helmberger (1995) showed that there was an approximately isotropic zone within a range of about $150 \mathrm{~km}$ in the top part of the inner core by analyzing the travel times of seismic body waves, however, Durek and Romanowicz (1999) presented that there should be strong anisotropy in the top part of the inner core to explain the known anomalous splitting of some EFO modes. Song and Helmberger (1998) provided some seismic evidences for the inner transition zone and pointed out a velocity discontinuity layer separating an isotropic upper inner core from an anisotropic lower inner core. Deuss (2008) discussed the shear wave velocity discrepancy between seismic data and mineral physical values in the inner core and considered that there probably existed the fluid inclusions in the inner core. Sun and Song (2008) did the three-dimensional tomography inversion for the anisotropy of the Earth's inner core and provided an inner core model with strong hemispherical and depth variation.

On the base of the Earth's displacement field, Gilbert and Masters calculated the distributions of elastic-wave energy density of EFO modes along the radius within the Earth and made the MINOS program (see http://stuplots.geophys.uni-stuttgart.de/ $\sim$ plots/Modes/modes.html). The elastic-wave energy density distribution consists of the compressive and shear energy density distribution of EFO modes. This kind of elastic-wave energy distribution was usually called the kernel functions of EFO modes, because they directly reflected the sensitivity of EFO modes to the elastic parameters of media in the different depth in the Earth. Those EFO modes sensitive to the elastic wave velocity in the inner core were usually called as the inner-core-sensitive modes, and they are still divided into the shear-wave-sensitive modes and the compressive-wave-sensitive modes. We checked 13 inner-core-sensitive modes consisting of eight shear wave sensitive modes $\left({ }_{3} \mathrm{~S}_{2},{ }_{2} \mathrm{~S}_{2},{ }_{10} \mathrm{~S}_{2},{ }_{5} \mathrm{~S}_{1},{ }_{4} \mathrm{~S}_{7},{ }_{5} \mathrm{~S}_{10}\right.$, ${ }_{7} \mathrm{~S}_{13}$ and $\left.{ }_{9} \mathrm{~S}_{4}\right)$ and five compressive wave sensitive modes $\left({ }_{4} \mathrm{~S}_{0},{ }_{3} \mathrm{~S}_{0},{ }_{8} \mathrm{~S}_{1},{ }_{13} \mathrm{~S}_{2}\right.$ and $\left.{ }_{13} \mathrm{~S}_{3}\right)$. The probe depth$\mathrm{s}$ of the shear-wave-sensitive modes and those of the compressive-wave-sensitive modes are listed in Tables 3 and 4 , respectively, and these parameters are approximately estimated on the basis of computation results of the MINOS program. We have calculated the relative deviation of every checked sensitive modes (Figures 2a and 3a). The relative deviations of the checked sensitive

Table 3 Probe depth of S wave inner-core-sensitive modes

\begin{tabular}{cccc}
\hline Mode & $\begin{array}{c}\text { Radius(enpk) } \\
/ \mathrm{km}\end{array}$ & $\begin{array}{c}\text { Radius1(halfpk) } \\
/ \mathrm{km}\end{array}$ & $\begin{array}{c}\text { Radius2(halfpk) } \\
/ \mathrm{km}\end{array}$ \\
\hline${ }_{9} \mathrm{~S}_{4}$ & 1115 & 929 & $1221.5^{*}$ \\
${ }_{7} \mathrm{~S}_{13}$ & 1104 & 987 & 1151 \\
${ }_{5} \mathrm{~S}_{10}$ & 1070 & 925 & 1150 \\
${ }_{4} \mathrm{~S}_{7}$ & 1030 & 822 & 1104 \\
${ }_{5} \mathrm{~S}_{1}$ & 940 & 580 & $1221.5^{*}$ \\
${ }_{10} \mathrm{~S}_{2}$ & 880 & 517 & 1198 \\
${ }_{2} \mathrm{~S}_{2}$ & 760 & 370 & 1060 \\
${ }_{3} \mathrm{~S}_{2}$ & 710 & 355 & 1020 \\
\hline
\end{tabular}

Notes: Radius(enpk) is the radius at which the shear waves occupy the maximum energy density, radius1(halfpk) and radius2(halfpk) are the radii at which the shear waves occupy the half maximum energy density in the inner core for the $\mathrm{S}$ wave sensitive modes. These parameters are approximately estimated on the computation results of the MINOS program (http://stuplots.geophys.unistuttgart.de/ plots/Modes/modes.html). The values with * mark prefer to the boundary of the Earth's inner core (BIC) when Radius2(halfpk) are larger than BIC.

Table 4 Probe depth of $\mathrm{P}$ wave inner-core-sensitive modes

\begin{tabular}{cccc}
\hline Mode & $\begin{array}{c}\text { Radius(enpk) } \\
/ \mathrm{km}\end{array}$ & $\begin{array}{c}\text { Radius1(halfpk) } \\
/ \mathrm{km}\end{array}$ & $\begin{array}{c}\text { Radius2(halfpk) } \\
/ \mathrm{km}\end{array}$ \\
\hline${ }_{13} \mathrm{~S}_{3}$ & 1221.5 & 1036 & $1221.5^{*}$ \\
${ }_{13} \mathrm{~S}_{2}$ & 1221.5 & 936 & $1221.5^{*}$ \\
${ }_{8} \mathrm{~S}_{1}$ & 1221.5 & 900 & $1221.5^{*}$ \\
${ }_{3} \mathrm{~S}_{0}$ & 850 & 478 & $1221.5^{*}$ \\
${ }_{4} \mathrm{~S}_{0}$ & 690 & 319 & 1036 \\
\hline
\end{tabular}

Notes: Radius(enpk) is the radius at which the compressive waves occupy the maximum energy density, radius1(halfpk) and radius2(halfpk) are the radii at which the compressive waves occupy the half maximum energy density in the inner core for the $\mathrm{P}$ wave sensitive modes. These parameters are approximately estimated on the computation results of the MINOS program (http://stuplots.geophys.unistuttgart.de/ p plots/Modes/modes.html). The values with * mark prefer to the boundary of the Earth's inner core (BIC) when Radius2(halfpk) are larger than BIC. 

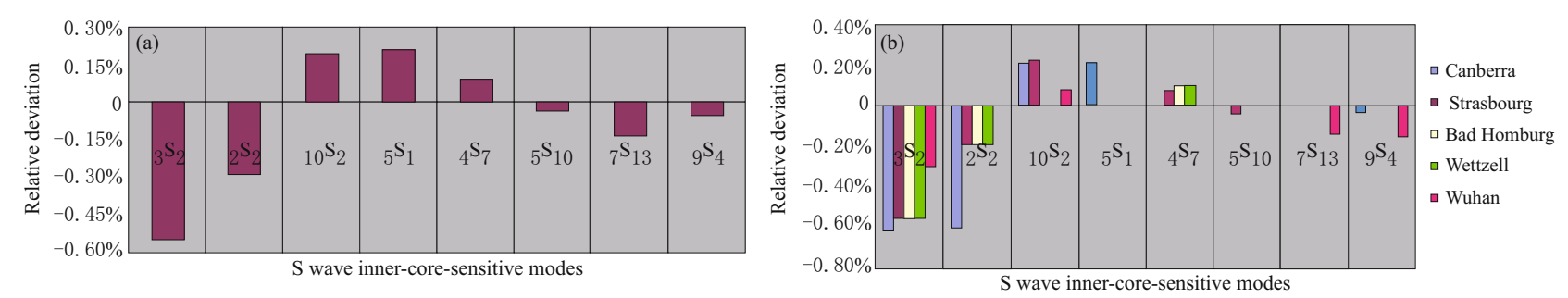

Figure 2 Relative deviations of some S wave inner-core-sensitive modes from PREM model. (a) Relative deviations of the checked EFO modes. (b) Relative deviations of five groups of EFO modes observed separately by five stations.
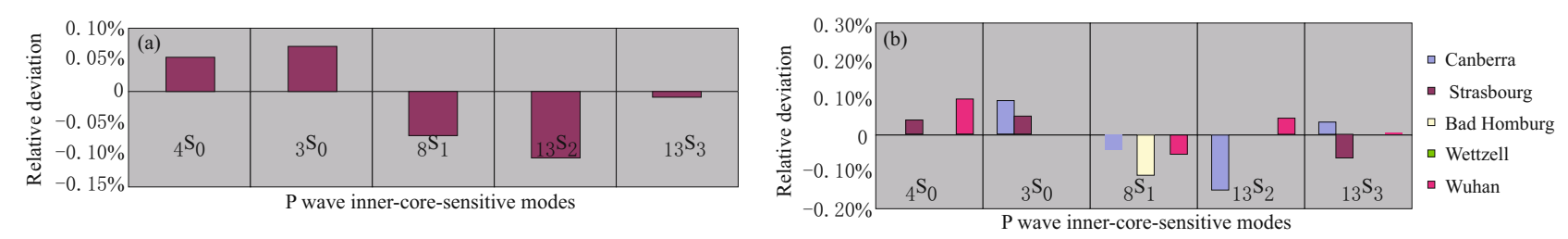

Figure 3 Relative deviations of some $\mathrm{P}$ wave inner-core-sensitive modes from PREM model. (a) Relative deviations of the checked EFO modes. (b) Relative deviations of five groups of EFO modes observed separately by five stations.

modes at every station are also computed to investigate the lateral variation of the inner core (Figures $2 \mathrm{~b}$ and $3 \mathrm{~b})$. The relative deviations of those sensitive modes reflect the variations of elastic wave velocity in the probe depths zone in the inner core. PREM is a classic Earth model based on many kinds of observation data, so we may discuss the structure of the Earth's inner core by analyzing the relative deviations of some sensitive modes from the PREM model.

Analysis on the relative variation of the shear wave velocity shown in Figure 2a and the probe depths of relevant sensitive modes listed in Table 3 both indicated that the inner core may be divided into three layers, i.e., the upper inner core (UIC) with lower shear velocity, the middle inner core (MIC) with higher shear velocity and the inner inner core (IIC) with very lower shear velocity. The lower boundary of UIC is about at the position between the probe depth of ${ }_{5} \mathrm{~S}_{10}$ mode $(151 \mathrm{~km})$ and the probe depth of ${ }_{4} \mathrm{~S}_{7}$ mode $(191 \mathrm{~km})$, therefore we adopt the average depth of $171 \mathrm{~km}$ below the boundary of the inner core, which is approximately compatible with the inner transition zone reported by Song and Helmberger (1998). There are some differences between the three sensitive modes $\left({ }_{5} \mathrm{~S}_{10},{ }_{7} \mathrm{~S}_{13},{ }_{9} \mathrm{~S}_{4}\right)$ observed at different GGP station shown in Figure $2 \mathrm{~b}$ and it mirrors that UIC still occupies some anisotropy and is similar to the former observation results based on the seismic EFO data (Durek and Romanowicz, 1999).

The top boundary of IIC is close to the position between the probe depth of ${ }_{2} \mathrm{~S}_{2}$ mode $(461 \mathrm{~km})$ and the probe depth of ${ }_{10} \mathrm{~S}_{2}$ mode $(341 \mathrm{~km})$, we accepted the average depth of about $400 \mathrm{~km}$ below the boundary of the inner core, which is larger $220 \mathrm{~km}$ than the distinct IIC described by Sun and Song (2008). Of course, the wide kernel functions of ${ }_{2} \mathrm{~S}_{2}$ and ${ }_{3} \mathrm{~S}_{2}$ modes also decrease the accuracy of the checked boundary. A clear difference between the observed ${ }_{2} \mathrm{~S}_{2}$ modes at different GGP stations (Figure $2 \mathrm{~b}$ ) reflects strong lateral anisotropy in the lower inner core and there is a very lower shear velocity observed at Canberra station, which may be related to the strong hemispherical variation in the inner core presented by Sun and Song (2008). MIC has a higher shear velocity and this is possibly related to the anisotropy zone in the inner core pointed out by Sun and Song (2008).

According to the relative variation of the compressive wave velocity (Figure 3a) and the probe depths of relevant sensitive modes (Table 4), we divides the inner core into two layers including the upper layer with lower compressive velocity and the lower layer with higher compressive velocity. The boundary of the two layers is about at the position between the probe depth of ${ }_{3} \mathrm{~S}_{0}$ mode $(371 \mathrm{~km})$ and the probe depth of ${ }_{8} \mathrm{~S}_{1}$ mode $(321$ $\mathrm{km}$ ), and its average depth is about $346 \mathrm{~km}$ below the boundary of the inner core, which is close to the upper boundary of IIC determined by the shear wave sensitive modes. As there are not enough compressive sensitive modes, the upper layer can not be divided into two lay- 
ers again like the shear sensitive modes. In fact, the lower layer occupies higher compressive wave velocity in the inner core. However, an intriguing thing is that there is suddenly a very lower shear wave velocity zone in IIC, which may be related with the possible existence of fluid inclusions in the inner core (Deuss, 2008).

\section{Conclusions}

EFO phenomena provide a path independent of seismic body waves to investigate the Earth's structure, however experimental EFO mode serials were mainly checked by the long-period seismographs or spring gravimeters before. Total of 147 EFO modes including 43 fundamental modes, five radial modes and 99 harmonic modes were checked with the superconducting gravimeters in this paper, which provided the serials of newer experimental EFO modes and the valuable constraint on the Earth's deep structure.

By investigating the relative deviations of some experimental sensitive modes from PREM model, the Earth's inner core could be modeled as three layers and the upper layer is compatible with the inner transition zone reported by Song and Helmberger (1998) with the seismic body data. The inner core also occupies a kind of hemispherical variation like the presentation by Sun and Song (2008). An interesting fact is that there is a very lower shear velocity zone in the lower inner core, which possibly indicates the existence of fluid inclusions in the inner core suggested by Deuss (2008).

Acknowledgements We are grateful to the GGP groups for providing the SG observation datasets of the great Sumatra-Andaman earthquake. We applied and referenced to the computation results of MINOS program and expressed our thanks for their study. The research was supported jointly by the National Natural Science Foundation of China (Nos. 40974046, 90814009 and 40730316), the Natural Science Foundation of Hubei Province (No. 2008CDB389) and the Knowledge Innovation Project of Chinese Academy of Sciences (No. KZCX2-YW-133). We sincerely appreciate the two anonymous reviewers for their suggestions.

\section{References}

Anderson O L (1995). Mineral physics of iron and of the core. Rev Geophys 33(Suppl): 429-441.

Banka D, Jentzsch G and Crossley D (1998). Investigations of superconducting gravimeter records in the frequency range of the free oscillations of the Earth - the noise magnitude. In: Proc. 13th Symp on Earth Tides. Obser- vatoire Royal de Belgique, Brussels, 641-648.

Beghein C and Trampert J (2003). Robust normal mode constraints on inner-core anisotropy from model space search. Science 299: 552-555.

Benioff H, Press F and Smith S (1961). Excitation of the free oscillations of the earth by earthquake. $J$ Geophys Res 66(2): 605-619.

Birch F (1964). Density and composition of mantle and core. $J$ Geophys Res 69(20): 4377-4 388.

Creager K C (1992). Anisotropy of the inner core from differential travel-times of the phases PKP and PKIKP. Nature 356: 309-314.

Crossley D J and Hinderer J (1995). Global Geodynamics Project-GGP: Status report 1994. In: Proc. 2nd Workshop 'Non-tidal Gravity Changes: Intercomparison Between Absolute and Superconducting Gravimeters'. Cahiers du Centre Européen de Géodynamique et de Séismologie, Walferdange, Luxembourg, 11: 244-274.

Derr J S (1969). Internal structure of the earth inferred from free oscillations. J Geophys Res 74(22): 5 202-5 220.

Deuss A (2008). Normal mode constraints on shear and compressional wave velocity of the Earth's inner core. Earth Planet Sci Lett 268(3-4): 364-375.

Ding Y R (1998). Processing Method of Astronomical Data. Nanjing University Press, Nanjing, 190-202 (in Chinese).

Durek J J and Romanowicz B (1999). Inner core anisotropy inferred by direct inversion of normal mode spectra. Geophys J Int 139(3): 599-622.

Dziewonski A M and Anderson D L (1981). Preliminary reference Earth model. Phys Earth Planet Interi 25(4): 297-356.

Dziewonski A M and Gilbert F (1972). Observations of normal modes from 84 recordings of the Alaskan earthquake of 1964 March 28. Geophys J R astr Soc 27: 293-446.

Farrell W E (1972). Deformation of the Earth by surface loads. Rev Geophys Space Phys 10(3): 761-797.

Fu C Y, Chen Y T and Qi G Z (1985). Foundation of Geophysics. 2nd edition. Science Press, Beijing, 347-368 (in Chinese).

Garland G D (1979). Introduce to Geophysics (Mantle, Core and Crust). 2nd edition. W. B. Saunders Company, Toronto, 123-133.

He X and Tromp J (1996). Normal mode constraints on the structure of the Earth. J Geophys Res 101(9): 20053 20082.

Ishii M, Tromp J, Dziewonski A M and Ekstrom G (2002). Joint inversion of normal mode and body wave data for inner core anisotropy: laterally homogeneous anisotropy. $J$ Geophys Res 107(B12): 2 379-2 395.

Laske G and Masters G (1999). Limits on differential rotation of the inner core from an analysis of the Earth's free oscillations. Nature 402(6757): 66-69.

Lei X E (2003). Study and Check of the FCN Parameters and the Earth's Free Oscillations. Ph D Dissertation. Gradu- 
ate University of the Chinese Academy of Sciences, Beijing, 170pp (in Chinese with English abstract).

Lei X E, Sun H P, Hsu H T and Shi Y L (2007). Check of Earth's free oscillations excited by Sumatra-Andaman large earthquake and discussions on the anisotropy of inner core. Science in China (Series D) 50(6): 909-917.

Lei X E, Xu H Z and Sun H P (2002a). Preliminary results of the Earth's free oscillations after Peru earthquake observed using a SG in China. Bull D'infor Marees Terrestres 135: 10 713-10 715 .

Lei X E, Xu H Z and Sun H P (2002b). Check of free oscillation signal with SG data. Chinese Science Bulletin 47(8): 1573-1578.

Masters G and Gilbert F (1981). Structure of the inner core inferred from observations of its spheroidal shear modes. Geophys Res Lett 8(6): 569-571.

Merriam J B (1992). Atmospheric-pressure and gravity. Geophys J Int 109(3): 488-500.

Morelli A, Dziewonski A M and Woodhouse J H (1986). Anisotropy of inner core inferred from PKIKP travel times. Geophys Res Lett 13(13): 1545-1 548.

Ness N R, Harrison C T and Slichter L B (1961). Observation of the free oscillation of the earth. $J$ Geophys Res 66(2): 621-629.

Neumeyer J, Bathelmes F, Combrinck L, Dierks O and Fourie P (2002). Analysis results from the SG registration with the dual sphere superconducting gravimeter at SAGOS (South Africa). Bull D'infor Marees Terrestres 135: $10607-10616$.

Niebauer T M (1988). Correcting gravity measurements for the effects of local air-pressure. J Geophys Res 93(B7): 7989-7 991.

Park J, Song T R A, Tromp J, Okal E, Stein S, Roult G, Clevede E, Laske G, Kanamori H, Davis P, Berger J, Braitenberg C, Van Camp M, Lei X, Sun H P, Xu H Z and Rosat S (2005). Earth's free oscillations excited by the 26 December 2004 Sumatra-Andaman earthquake. Science 308(5725): 1 139-1 144.

Poupinet G, Pillet R and Souriau A (1983). Possible heterogeneity of the Earth's core deduced from PKIKP travel times. Nature 305: 204-206.

Rosat S, Hinderer J, Crossley D and Boy J P (2004). Performance of superconducting gravimeters from long-period seismology to tides. J Geodyn 38(3-5): 461-476.

Rosat S, Sato T, Imanishi Y, Hinderer J, Tamura Y and McQueen H (2005). High-resolution analysis of the gravest seismic normal modes after the $2004 M_{\mathrm{W}}=9$ Sumatra earthquake using superconducting gravimeter data. Geophys Res Lett 32: L13304.

Shearer P M (1994). Constraints on inner core anisotropy from PKP(DF) travel-times. J Geophys Res 99(B10): 19647-19657.
Shearer P M and Masters G (1990). The density and shear velocity contrast at the inner core boundary. Geophys $J$ Int 102(2): 491-498.

Song X D and Helmberger D V (1995). Depth dependence of anisotropy of Earth's inner core. J Geophys Res 100(B7): 9 805-9816.

Song X D and Helmberger D V (1998). Seismic evidence for an inner core transition zone. Science 282(5390): 924927 .

Stein S and Okal E A (2005). Speed and size of the Sumatra earthquake. Nature 434(7033): 581-582.

Stixrude L and Cohen R E (1995). High-pressure elasticity of iron and anisotropy of Earth's inner core. Science 267(5206): 1972-1975.

Sun H P (1997). Atmospheric pressure and gravity. Chinese Science Bulletin 42(1): 1712-1 719.

Sun H P, Chen X D, Hsu H T and Wang Y (2001). Accurate determination of calibration factor for tidal gravity observation of a GWR-superconducting gravimeter. Acta Seismologica Sinica 14(6): 692-700.

Sun X L and Song X D (2008). The inner inner core of the Earth: Texturing of iron crystals from three-dimensional seismic anisotropy. Earth Planet Sci Lett 269(1-2): 5665.

Tamura Y (1987). A harmonic development of the tidal generating potential. Bull Inf Marées Terrestres 99: 68136855.

Tromp J (1993). Support for anisotropy of the Earth's inner core from free oscillations. Nature 366: 678-681.

Van Camp M (1999). Measuring seismic normal modes with the GWR C021 superconducting gravimeter. Phys Earth Planet Interi 116(1-4): 81-92.

Widmer R, Masters G and Gilbert F (1991). Spherically symmetric attenuation within the Earth from normal mode data. Geophys J Int 104(3): 541-553.

Woodhouse J H, Giardini D and Li X D (1986). Evidence for inner core anisotropy from free oscillations. Geophys Res Lett 13: 1549-1552.

Xi Q W (1998). The surface spherical harmonics and the development of tidal generating potential. Acta Seismologica Sinica 11(4): 511-513.

Xu H Z, Sun H P, Xu J Q and Tao G X (2000). International tidal gravity reference values at Wuhan station. Science in China (Series D) 43(1): 77-83.

Xu J Q, Hao X H and Sun H P (1999). Influence of atmospheric pressure on tidal gravity at Wuhan station. Acta Geophysica Sinica 28(1): 21-27 (in Chinese with English abstract).

Zürn W and Widmer R (1995). On noise reduction in vertical seismic records below $2 \mathrm{mHz}$ using local barometric pressure. Geophys Res Lett 22(24): 3 537-3 540. 